\title{
TOLERABILITY, COMPLIANCE, AND PRODUCT EVALUATION OF A PRE-THICKENED ORAL NUTRITIONAL SUPPLEMENT FOR DISEASE RELATED MALNUTRITION IN PATIENTS WITH DYSPHAGIA
}

\author{
T. Dennehy' ${ }^{1}$ F. Veldkamp², M. Lansink ${ }^{3}$, R.J. Schulz ${ }^{4}$
}

\begin{abstract}
Background: Oropharyngeal dysphagia is a prevalent risk factor for malnutrition in older patients and both conditions are related to poor outcome. For the management of (risk of) malnutrition in patients with oropharyngeal dysphagia pre-thickened oral nutritional supplements are available. Objective: The objective of the study is to describe tolerance parameters (stool frequency and incidence and intensity of gastrointestinal symptoms), study product intake (compliance), product appreciation and product properties of a pre-thickened oral nutritional supplement compared to a manually-thickened standard oral nutritional supplement. Design: A randomized, open label, controlled, parallel group study. Setting: participants were recruited through nine general practices in Ireland, one nursing home in The Netherlands, and one hospital in Germany. Participants: Fifty patients requiring oral nutritional support (twenty-four of fifty cases $(48 \%)$ with dysphagia) were divided into two groups: test group ( $\mathrm{N}=27)$ and control group $(\mathrm{N}=23)$. Intervention: During four weeks the test group received a ready-to-use, low volume $(125 \mathrm{~mL})$, and energy dense pre-thickened oral nutritional supplement, and the control group a manually-thickened iso-caloric oral nutritional supplement $(200 \mathrm{~mL})$ with a similar viscosity. Measurements: Compliance and stool frequency were recorded daily, evaluation of the product appreciation and properties and gastrointestinal tolerability were assessed with questionnaires. Results: Incidence and intensity of gastrointestinal symptoms was not statistically different between groups. Pre-thickened oral nutritional supplement scored significantly better on compliance in week $4(\mathrm{p}=0.019)$, on thickness appreciation by patients $($ day $14, \mathrm{p}=0.035)$ and on product properties evaluation by carers (appearance, preparation time, ease of preparation and change in thickness, all $\mathrm{p}<0.001$ ) compared to the manually-thickened ONS. Conclusion: These results substantiate the use of pre-thickened oral nutritional supplement for the dietary management of patients in need of nutritional support and with oropharyngeal dysphagia.
\end{abstract}

Key words: Pre-thickened ONS, dysphagia, gastro-intestinal (GI) tolerability, compliance, product properties.

\section{Introduction}

Oropharyngeal dysphagia (OD) is a clinical symptom, defined by a difficulty or inability to form or to move the alimentary bolus safely from the mouth to the oesophagus. OD may cause choking, aspiration, aspiration pneumonia, malnutrition, dehydration, and death due to complications (1-3). Swallowing disorders are prevalent in different conditions/diseases e.g. aging, stroke, head and neck cancer, and Alzheimer's and other neurological disorders. OD can considerably affect the patient's quality of life (4).

Modifying the texture of foods and the viscosity of fluids by thickeners is considered to be one of the basic compensatory interventions in the management

1. Bank House Surgery, Cork, Ireland; 2. TOPAZ Overduin, Katwijk, The Netherlands; 3. Danone Nutricia Research, Utrecht, The Netherlands; 4. Department of Geriatric Medicine, St Marien Hospital, Cologne

Corresponding Author: M. Lansink, Nutricia Research, Uppsalalaan 12, 3584 CT Utrecht, The Netherlands T: +31 30 2095000, M: +31 6 27847940, E-mail address: mirian.lansink@nutricia.com of OD by practitioners in the field of speech and language therapy (SLT), nurses and dietitians (5). The terminology used to describe the consistency of thickened liquids and the number of levels/stages varies widely between countries. For instance until last year, in the UK the British Dietetic Association (BDA) system was used to describe the viscosity levels: stage 1, 2, 3 (6). The terminology has recently been evaluated by the International Dysphagia Diet Standardization Initiative (IDDSI) and European Society for Swallowing Disorders (ESSD). IDDSI published a framework for a global standardised terminology and definitions to describe texture modified foods and thickened liquids, consisting of a continuum of 8 levels (0-7) (7). ESSD concluded that there is evidence for increasing viscosity to reduce the risk of airway invasion in a viscosity-dependent manner and this is a valid management strategy for OD (8). In addition, to improve the safety of patients the ESSD is developing a labelling system of viscosity levels for fluid thickening (based on SI units of viscosity (mPa.s) at 50s-1 and $25^{\circ} \mathrm{C}$, effect of salivary amylase and shear thinning on 
viscosity).

Patients with dysphagia on a texture modified diet usually do not reach their dietary requirements (9). Inferior sensory and/or nutritional qualities of texture modified diets may play a role in this and use of modified texture foods is highly associated with undernutrition (10, 11). Therefore, nutritional intake should be facilitated and increased by enriching meals and/or using ONS $(12,13)$. It is important for OD patients that ONS have the right thickness; however, the intra and inter subject variation and lack of proper training of carers and users in the preparation of thickened drinks and/or pureed meals may lead to variation in the viscosity of fluids provided to dysphagia patients $(14,15)$. This may negatively influence food and fluid intake, but also safety of swallowing (16). For dysphagia patients, pre-thickened, ready to use ONS are available, but also standard ONS manually thickened to the prescribed viscosity stage are used in clinical practice. The success of nutritional support depends not only on the supplied energy and nutrients, but also on compliance by the users, which might be positively affected by the ONS energy-density (17).

In the present study four weeks use of a pre-thickened, high energy dense, low volume ONS $(125 \mathrm{~mL}$, test product) in patients in need of nutritional support was compared to that of a manually thickened, isocaloric ONS (200 mL, control product) with respect to GI tolerability, compliance, subjective evaluation of product appreciation and product properties.

\section{Methods}

\section{Subjects}

Patients in need of nutritional support, as prescribed by a health-care professional, were included in the study over a period of 1 year. Inclusion criteria were age of $\geq$ 18 years, a prescription for oral nutritional support of $\geq 300 \mathrm{kcal} /$ day of energy enriched sip feed or, in case of new users, a Malnutrition Universal Screening Tool (MUST) score of 1 or higher (malnutrition risk for score 0,1 , and $\geq 2$ is low, medium and high, respectively), a requirement for oral nutritional support for at least four weeks and written informed consent. Half of the patients should have been diagnosed with dysphagia and require thickened drinks with a stage 1 consistency according to the BDA classification. Exclusion criteria were participation in any other study involving investigational or marketed products concomitantly or within two weeks prior to entry into the study, requirement of oral nutritional support other than energy enriched ONS (e.g. high protein sip feeds, disease specific sip feeds), known lactose intolerance or galactosaemia, major hepatic or renal dysfunction, known inflammatory bowel diseases, having an ileostomy or colostomy, strong dislike of the flavours to be tested (strawberry and vanilla) and investigator's uncertainty about the willingness or ability of the subject to comply with the study protocol.

\section{Study design}

This randomized, prospective, open label, controlled, parallel-group multi-centre study (Netherlands Trial Register: NTR1643) was performed with nine general practices in Ireland, one nursing home in The Netherlands, and one hospital in Germany. The study is compliant with the ethical guidelines of the Declaration of Helsinki and approved by the ethics committees of the local sites.

At baseline, subjects were randomly assigned to either of the study groups, using minimization for ONS prescription (2 options: 1 serving/day, 2 servings/day) and requiring thickened drinks (2 options: yes, no). A hybrid minimization procedure was chosen (18).

Allocation to study groups started randomly and continued as long as balanced distribution was maintained for ONS prescription and requirement of thickened drinks. Since the control product was different in volume from the test product and, unlike the test product, the control product required thickening, it was not possible to blind the subjects, carers and physicians.

Test product was a pre-thickened, low volume (125 $\mathrm{mL}$ ) energy-dense, fibre-enriched ONS with amylaseresistance features (Nutilis Complete Stage 1, Nutricia N.V. Zoetermeer, The Netherlands) and the control product an isocaloric standard volume $(200 \mathrm{~mL})$ fibreenriched ONS (Fortisip Multi-fibre, Nutricia N.V. Zoetermeer, The Netherlands) manually thickened with commercially available thickeners. The product compositions are shown in table 1 . The pre-thickened product had a targeted viscosity of $450 \mathrm{mPa}$.s determined at a shear rate of $50 \mathrm{~s}-1$ and a temperature of $20^{\circ} \mathrm{C}$, and it corresponds to IDDSI level 3 and to stage 1 of the former BDA $(6,7)$. Viscosities of the control was thickened at stage 1 BDA consistency with the available thickener used in each location.

Table 1

Product composition

\begin{tabular}{lcc}
\hline & Test & Control $^{*}$ \\
\hline Volume (mL) & 125 & 200 \\
Energy (kcal) & 306 & 300 \\
Energy density (kcal/ml) & 2.45 & 1.50 \\
Protein (g) & 12 & 12 \\
Fat (g) & 11.6 & 11.6 \\
Carbohydrate (g) & 36.4 & 36.8 \\
Dietary fibre (g) & 4 & 4.6 \\
Sodium (mg) & 112 & 178 \\
Osmolarity (mOsmol/L) & 495 & 455 \\
\hline *The control product is thickened with the thickening powder to a stage 1 \\
consistency (BDA classification).
\end{tabular}


Study endpoints were stool frequency and incidence and intensity of each GI symptom per time point, study product compliance, patients' evaluation on product appreciation and carer's evaluation on the properties of products. Stool frequency was assessed daily, and incidence and intensity of GI symptoms were assessed during the baseline period and at day 14 and day 28 of the study. Compliance was assessed daily, and it was defined as having a mean intake of at least $75 \%$ of the recommended volume during the intervention period. Patients' evaluation on product appreciation was assessed at day 14 and 28 after consuming the product, and carer's evaluation on product properties was collected at the end of the study. Occurrence of adverse events and medication use was recorded throughout the study period. Information on incidence and intensity of GI symptoms in the past 2 days was collected using a 4-point scale (absent (0), mild (1), moderate (2), severe (3)). This questionnaire was completed by the subject or carer and GI symptoms scored were: nausea, vomiting, diarrhoea, constipation, abdominal distension (bloating), belching, and flatulence. Product appreciation scored by the subjects able to complete the questionnaire were: taste, sweetness, mouth feel, thickness, aftertaste, and mouth feel after swallowing using a hedonic 7-point scale (ranging from "I like it very much" to "I dislike it very much"). Product properties scored by carers were: appearance, preparation time, ease of preparation, and change in thickness using a hedonic 5-point scale. Safety was determined by evaluating adverse events (AEs) and serious adverse events (SAEs).

\section{Statistics}

Subject characteristics at initial assessment were summarised per group. Group comparisons for stool frequency, incidence and intensity of GI symptoms, number of subjects who were compliant and number of subjects with AEs, product appreciation (clustered as negative (1-3), neutral (4) or positive (5-7)) and carer's evaluation of product properties were analysed using Fisher's exact test. A p-value of $<0.05$ (two-sided) were considered statistically significant. In the event of missing data, these data points were treated as 'missing' and not included as valid data points in the analyses. No correction for multiplicity has been applied. For statistical analysis the results of the intention to treat (ITT) population $(\mathrm{N}=50)$ were used. Statistical analysis was performed using SPSS version 15.0 for Windows.

\section{Results}

\section{Patients}

Fifty patients were included in the study, 27 in the test group and 23 in the control group. Twelve patients did not complete the study: 6 in the test group and 6 in the control group. Reasons for early termination included dislike of the study product, AEs and discharge of patients. Table 2 summarizes the demographics and patient characteristics. Thirteen of twenty-seven subjects in the test group and eleven of twenty-three subjects in the control group (48\% of the patients in both groups) had swallowing problems and required thickened drinks with a BDA stage 1 consistency (table 2).

Table 2

Demographics and characteristics of randomised patients

\begin{tabular}{|c|c|c|}
\hline & Test $(n=27)$ & Control $(n=23)$ \\
\hline Age (years) mean $\pm S D$ & $80.4 \pm 10.0$ & $67.4 \pm 19.3$ \\
\hline Male & $14(52)$ & $11(48)$ \\
\hline Female & $13(48)$ & $12(52)$ \\
\hline $\mathrm{BMI}(\mathrm{kg} / \mathrm{m} 2)$ mean $\pm \mathrm{SD}^{*}$ & $19.9 \pm 2.9$ & $20.9 \pm 3.2$ \\
\hline \multicolumn{3}{|l|}{ MUST scoret } \\
\hline Score 1 & $14(52)$ & $11(48)$ \\
\hline Score 2 & $0(0)$ & $2(9)$ \\
\hline Score 3 & $13(48)$ & $10(43)$ \\
\hline \multicolumn{3}{|l|}{ Prescription } \\
\hline 1 bottle & $20(74)$ & $16(70)$ \\
\hline$\geq 2$ bottles & $7(26)$ & $7(30)$ \\
\hline \multicolumn{3}{|l|}{ Required thickened drinks? } \\
\hline Yes & $13(48)$ & $11(48)$ \\
\hline No & $14(52)$ & $12(52)$ \\
\hline \multicolumn{3}{|c|}{$\begin{array}{l}\text { Data are given in } \mathrm{n}(\%) \text { unless otherwise indicated; }{ }^{*} \text { BMI (Body Mass Index): } \\
<18.5 \text { (underweight), } 18.5-24.9 \text { (normal weight), } 25-29.9 \text { (overweight), }>30 \\
\text { (obesity). BMI at baseline was available for } 48 \text { subjects: test }(n=26) \text { and } \\
\text { control ( } \mathrm{n}=22 \text { ); }+ \text { MUST score: } 0 \text { (low risk of malnutrition), } 1 \text { (medium risk of } \\
\text { malnutrition), } \geq 2 \text { (high risk of malnutrition) }\end{array}$} \\
\hline
\end{tabular}

\section{Stool frequency and incidence and intensity of GI symptoms}

There were no significant differences in stool frequency between test and control group (table 3 ) nor for the dysphagia subgroup (data not shown), and the majority of subjects had 0-1 stools per day. The majority of GI symptoms were absent or mild for both groups. No GI symptoms were reported as severe in the test group. In the control group there was one incidence of severe constipation and one incidence of severe diarrhoea at baseline. At day 14, one incidence of severe belching and two incidences of severe flatulence were reported. However, no significant differences in incidence and intensity per GI symptom were observed between the groups either at baseline, day 14 or day 28; neither for the total group nor for the dysphagia subgroup (data not shown).

\section{Study product compliance}

Twenty of twenty-seven subjects in the test group and sixteen of twenty-three subjects in the control 
Table 3

Comparison of outcome parameters

\begin{tabular}{|c|c|c|c|c|c|}
\hline & \multicolumn{2}{|l|}{ Test } & \multicolumn{2}{|l|}{ Control } & p-value \\
\hline \multicolumn{6}{|l|}{ Primary endpoint } \\
\hline \multicolumn{6}{|l|}{ Mean stool frequency ${ }^{*}(\mathrm{n}(\%))$} \\
\hline \multicolumn{6}{|l|}{$(0-1 /$ day $/>1-2 /$ day $/>2 /$ day $)$} \\
\hline Baseline & \multicolumn{2}{|c|}{$13(81.3) / 3(18.8) / 0(0.0)$} & \multicolumn{2}{|c|}{$14(82.4) / 3(17.6) / 0(0.0)$} & $1.000+$ \\
\hline Week 2 & \multicolumn{2}{|c|}{$16(72.7) / 6(27.3) / 0(0.0)$} & \multicolumn{2}{|c|}{$17(77.3) / 4(18.2) / 1(4.5)$} & $0.721+$ \\
\hline Week 4 & \multicolumn{2}{|c|}{$17(81.0) / 4(19.0) / 0(0.0)$} & \multicolumn{2}{|c|}{$14(82.4) / 2(11.8) / 1(5.9)$} & $0.516+$ \\
\hline \multicolumn{6}{|l|}{ Secondary endpoints } \\
\hline (non)compliant subjects [>75\% intake](n (\%)) & non-compliant & compliant & non-compliant & compliant & \\
\hline Week 1 & $3(12.0)$ & $22(88.0)$ & $3(15.8)$ & $16(84.2)$ & $1.000+$ \\
\hline Week 2 & $4(19.0)$ & $17(81.0)$ & $3(16.7)$ & $15(83.3)$ & $1.000+$ \\
\hline Week 3 & $2(9.5)$ & $19(90.5)$ & $2(13.3)$ & $13(86.7)$ & $1.000+$ \\
\hline Week 4 & $0(0.0)$ & $21(100.0)$ & $4(28.6)$ & $10(71.4)$ & $0.019+$ \\
\hline Total & $4(16.0)$ & $21(84.0)$ & $5(23.8)$ & $16(76.2)$ & $0.711+$ \\
\hline
\end{tabular}

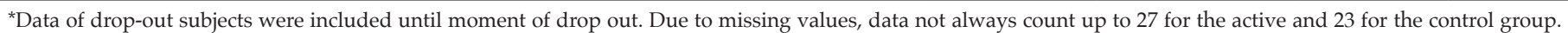

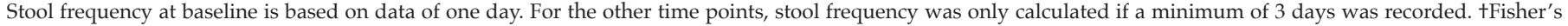
Exact test

group (about $70 \%$ of the subjects in both groups) were prescribed 1 bottle per day, the remaining patients being on a prescription of 2 or more bottles per day. No significant differences in the number of subjects who were compliant with study product intake were shown for the total intervention period between groups, twenty-one of twenty-five (84\%) subjects for the test product and sixteen of twenty-one $(76.2 \%)$ subjects for the control product, $\mathrm{p}=$ 0.711 (table 3), nor for the dysphagia subgroup. For week 4 a significantly higher percentage of subjects in the test group was compliant as compared to the control group, all the subjects in the test group $(n=21)$ vs ten of fourteen $(71.4 \%)$ subjects in the control group $\mathrm{p}=0.019$ (table 3). No differences of body weight between test and control group were found at different time points (baseline, day 14 , and day 28) $(\mathrm{p}>0.05)$.

\section{Patients' evaluation on product appreciation and carer's evaluation on product properties}

The questionnaire used to evaluate the product appreciation was filled in by 23 patients in the test group and 22 patients in the control group at day 14 and by 21 and 16 patients respectively at day 28 . No significant difference in five aspects (taste, sweetness, mouth feel, aftertaste, and mouth feel after swallowing) was found between groups. Thickness appreciation of the test product scored significantly higher compared to the control product at day $14(\mathrm{p}=0.035)$.

The questionnaire used to evaluate the product properties was filled in by 13-22 carers for the test product (appearance: $\mathrm{n}=22$, preparation time: $\mathrm{n}=13$, ease of preparation: $\mathrm{n}=15$ and change in thickness: $\mathrm{n}=18$ ) and 17 carers for the control product. For all four aspects significant differences were found between test and control product: carers indicated that product appearance $(\mathrm{p}=0.049)$, preparation time $(\mathrm{p}<0.001)$, ease of preparation $(\mathrm{p}<0.001)$ and stability of consistency $(\mathrm{p}<$ 0.001 ) were significantly better for the pre-thickened ONS compared with manually-thickened ONS (figure 1).

\section{(Serious) adverse events $(S(A E)$ s)}

No significant differences were shown in the number of subjects with one, two or more adverse events between groups. In the test group 22 AEs occurred in 11 patients and none of these was reported as related to the study product. In the control group 18 AEs were reported in 11 patients. One AE (vomiting) was possibly related to the control product and $3 \mathrm{AEs}$ in one person (diarrhoea, abdominal cramps, nausea) were classified by the investigator as definitely related to the control product. During the study 5 SAEs (femur fracture, cholecystitis, hospital admission (due to vomiting and diarrhoea), pneumonia and cardiac arrest) were reported in the test group. All were classified as not related to the study product according to the investigator. In the control group, no SAEs were described.

\section{Discussion}

Current study results showed that no significant differences in stool frequency and incidence and intensity of GI symptoms were observed between the test and control group. There was no significant difference in product compliance between groups for the overall period, but in week 4 the pre-thickened ONS had a significantly better compliance. Product appreciation evaluated by the patients was not significantly different between groups except for the thickness appreciation which scored significantly better for the test group. Product properties evaluated by the carers were 
Figure 1

Carer's evaluation of appearance, preparation time, ease of preparation, and change in thickness

A

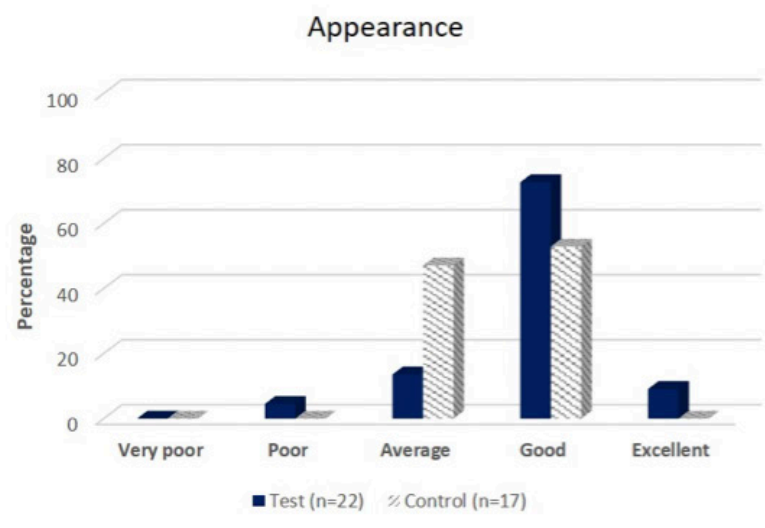

C

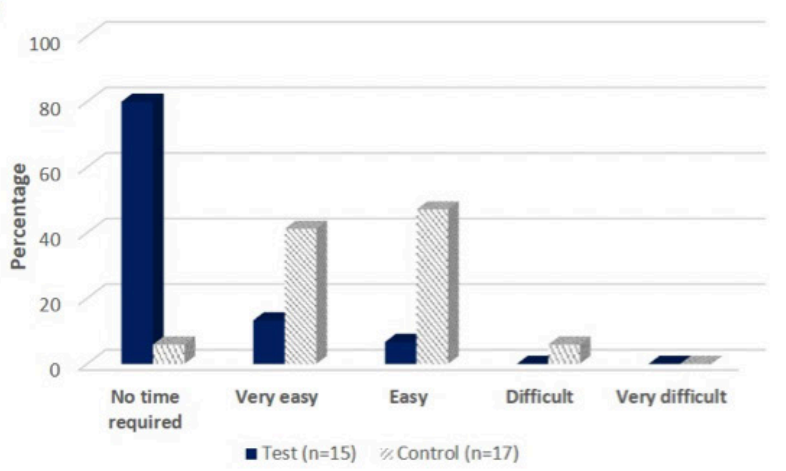

B

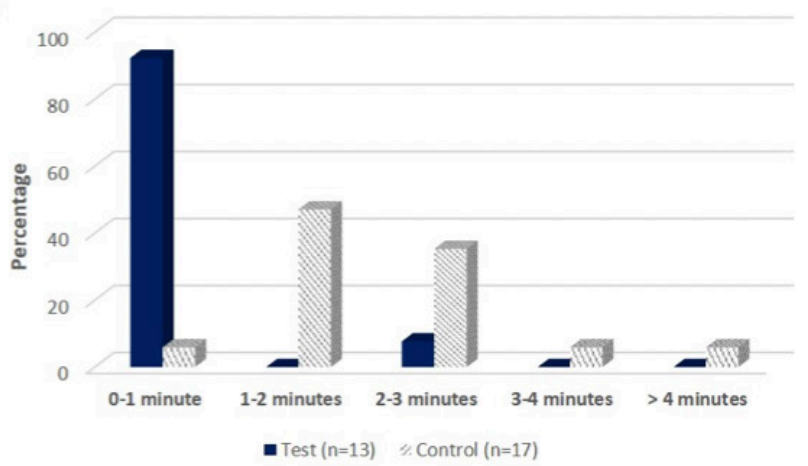

Change in thickeness

D

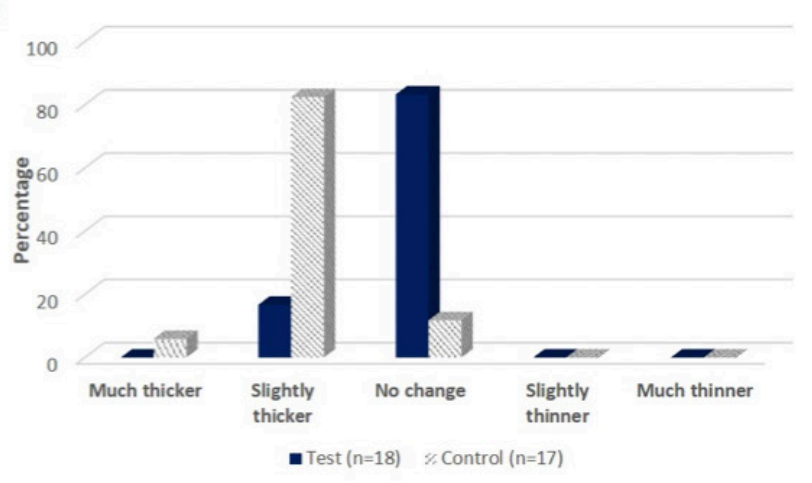

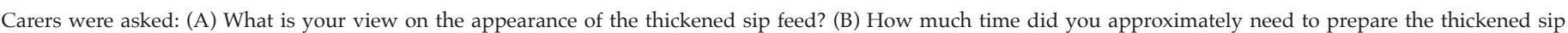

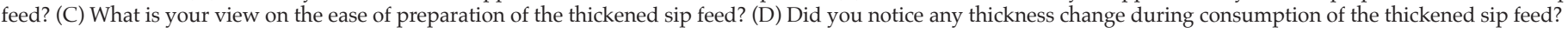

significantly better for the test product compared to the control product. Together these data substantiate the use of a pre-thickened ONS in patients with dysphagia.

For the total intervention period compliance was twenty-one of twenty-five (84\%) subjects in the test group and sixteen of twenty-one (76.2\%) subjects in the control group. These numbers are in line with the mean overall compliance to ONS of $78 \%$ previously reported in a systematic review (17). In this review, mean compliance to ONS in community studies was $80.9 \%$, whereas in the hospital setting an average $67.2 \%$ of the patients was compliant. In the current study, patients from both settings were included. Interestingly, in week 4 the compliance became significantly higher in the group consuming the energy dense pre-thickened test product as compared with the control group. This is in line with previous observations of significantly greater compliance and energy intakes with small volume, energy dense ONS compared with standard sip feeds $(17,19)$. Besides the low volume of the test product, pre-thickening of the ONS rather than manually thickening may also have an effect on compliance, as suggested by studies that found increased intake with pre-thickened drinks. In a small cross-over pilot study with 11 patients requiring thickened fluids it was suggested that the use of pre- thickened drinks (including milk, fruit juices, orange juice and tea) as compared to manually thickened drinks increases nutrients intake derived from drinks (20). And in a study with acute stroke patients with dysphagia, it was also shown that patients who received pre-thickened fluids drank almost $100 \%$ more than those on powder thickened fluids (21). It is not likely that in the current study a difference in appreciation of the products has played a role in the improved compliance in the test group in week 4, since the appreciation of the two ONS was not significantly different between groups except for thickness appreciation in favour of the pre-thickened ONS at day 14.

Body weight did not differ between test and control group at different time points (baseline, day 14, and day 28). Comparable nutritional value of test and control product and number of patients who were prescribed one bottle/day (about 70\%) or $\geq 2$ bottles / day (about 30\%) in the test and control group had probably contributed to this result.

The ready-to-use ONS was considered by the carers to have a better appearance, a shorter preparation time, was easier to prepare, and had a more stable consistency. Comparably, Kotecki and Schmidt reported that due to variability in the skill of the preparer and alteration 
in the viscosity of the product over time, commercially thickened liquids showed a more consistent viscosity than manually prepared thickened liquids (22). Based on their findings they conclude that the benefit of commercially thickened liquids is the potential for an improved quality of life for the patient with dysphagia. Other studies also reported that many patients are served manually thickened liquids that are too thick or too thin with respect to the target level of thickness $(14,23)$. In a study by MacLeod et al. 24 subjects with learning disabilities who required a modified texture diet with thickened fluids and did not meet their nutritional requirements, received pre-thickened ONS in a 4 week, open, noncontrolled, prospective observational pilot study. Results indicated that the pre-thickened ONS had advantages over existing treatment options as assessed by: ease of use $(71 \%)$, guaranteed a safer consistency $(71 \%)$, meets nutritional requirements $(58 \%)$ and compliance $(50 \%)$ (24). Together the findings above suggest that consistent viscosities and convenience of use appear to be important advantages of ready to use products as compared to manually thickened products.

Current study results indicate that GI tolerability of the pre-thickened ONS is similar to the manually-thickened ONS in patients in need of nutritional support. The GI symptoms recorded during this study were absent or mild during use of either test or control product. Both the test and control product of the current study contained a fibre blend consisting of a mixture of soluble and insoluble fibres (soy polysaccharides, cellulose, resistant starch, gum arabic, oligofructose and inulin). Previous studies in elderly patients and nursing home residents have shown that dietary fibres have positive effects on bowel movements and constipation $(25,26)$, which was also concluded in a systematic review (27).

In summary, the pre-thickened, small volume, energydense, fibre-enriched, ready-to-use ONS with amylaseresistant features was better appreciated by patients and/ or carers for its consistent viscosity and convenience of use, had a higher compliance in week 4 , and had a similar and good GI tolerability as compared to the manuallythickened, standard, isocaloric, fibre-enriched ONS. No safety issues based on AEs were reported. Therefore, these results substantiate the use of a pre-thickened ONS for the dietary management of patients in need of nutritional support with OD.

Funding: Sources of funding: this study was supported by Danone Nutricia Research. Danone Nutricia Research was responsible for the study: design and conduct, statistical analysis and interpretation of the results, preparation and approval of the manuscript.

Acknowledgements: All authors made substantial contributions to the conception and design of the study, or acquisition of data, or analysis and interpretation of data, in drafting the article, revising it critically, and have approved the final version submitted. We would like to thank Sonia Guida and Nick van Wijk for their contribution in revising the manuscript.

Conflict of interest disclosure: Declaration of interest: Mirian Lansink is an employee of Danone Nutricia Research and Tom Dennehy, Fleur Veldkamp, and
Ralf-Joachim Schulz declare no conflict of interest.

\section{References}

1. Almirall, J., et al., Oropharyngeal dysphagia is a risk factor for communityacquired pneumonia in the elderly. Eur Respir J, 2013. 41(4): p. 923-8.

2. Carrion, S., et al., Oropharyngeal dysphagia is a prevalent risk factor for malnutrition in a cohort of older patients admitted with an acute disease to a general hospital. Clin Nutr, 2015. 34(3): p. 436-42.

3. Ekberg, O., Dysphagia; diagnosis and treatment. Springer Berlin, 2012.

4. Ekberg, O., et al., Social and psychological burden of dysphagia: its impact on diagnosis and treatment. Dysphagia, 2002. 17: p. 139-146.

5. Cichero, J.A., Thickening agents used for dysphagia management: effect on bioavailability of water, medication and feelings of satiety. Nutr J, 2013. 12: p. 54.

6. Cichero, J.A., et al., The Need for International Terminology and Definitions for Texture-Modified Foods and Thickened Liquids Used in Dysphagia Management: Foundations of a Global Initiative. Curr Phys Med Rehabil Rep, 2013. 1: p. 280-291.

7. Cichero, J.A., et al., Development of International Terminology and Definitions for Texture-Modified Foods and Thickened Fluids Used in Dysphagia Management: The IDDSI Framework. Dysphagia, 2017. 32(2): p. 293-314.

8. Newman, R., et al., Effect of Bolus Viscosity on the Safety and Efficacy of Swallowing and the Kinematics of the Swallow Response in Patients with Oropharyngeal Dysphagia: White Paper by the European Society for Swallowing Disorders (ESSD). Dysphagia, 2016. 31(2): p. 232-49.

9. Wright, L., et al., Comparison of energy and protein intakes of older people consuming a texture modified diet with a normal hospital diet. J Hum Nutr Diet, 2005. 18(3): p. 213-9.

10. Keller, H., et al., Issues associated with the use of modified texture foods. J Nutr Health Aging, 2012. 16(3): p. 195-200.

11. Matta, Z., et al., Sensory characteristics of beverages prepared with commercial thickeners used for dysphagia diets. J Am Diet Assoc, 2006. 106(7): p. 1049-54

12. Wirth, R., et al., Oropharyngeal dysphagia in older persons - from pathophysiology to adequate intervention: a review and summary of an international expert meeting. Clin Interv Aging, 2016. 11: p. 189-208.

13. Volkert, D., et al., ESPEN guideline on clinical nutrition and hydration in geriatrics. Clin Nutr, 2018.

14. Smith, T.L., M.M. Sun, and J. Pippin, Research and professional briefs: characterizing process control of fluid viscosities in nursing homes. J Am Diet Assoc, 2004. 104(6): p. 969-71.

15. Glassburn, D.L. and J.F. Deem, Thickener viscosity in dysphagia management: variability among speech-language pathologists. Dysphagia, 1998. 13(4): p. 218-22.

16. RCSLT, Resource manual for commissioning and planning services for SLCN. Dysphagia. Royal College of Speech and Language Therapists., 2014.

17. Hubbard, G.P., et al., A systematic review of compliance to oral nutritional supplements. Clin Nutr, 2012. 31(3): p. 293-312.

18. Scott, N.W., et al., The method of minimization for allocation to clinical trials: a review. Controlled Clinical Trials, 2002. 23: p. 664-665.

19. Lombard, K., et al., Compliance of energy-dense, small volume oral nutritional supplements in the daily clinical practice on a geriatric ward--an observational study. J Nutr Health Aging, 2014. 18(7): p. 649-53.

20. McCormick, S.E., et al., The efficacy of pre-thickened fluids on total fluid and nutrient consumption among extended care residents requiring thickened fluids due to risk of aspiration. Age Ageing, 2008. 37(6): p. 714-5.

21. Whelan, K., Inadequate fluid intakes in dysphagic acute stroke. Clin Nutr, 2001. 20(5): p. 423-8.

22. Kotecki, S. and R. Schmidt, Cost and effectiveness analysis using nursing staff-prepared thickened liquids vs. commercially thickened liquids in stroke patients with dysphagia. Nursing Economics, 2010. 28 (2): p. 106-110.

23. J Garcia, E.C.I., M Clark, J Helverson, Z Matta, Quality of care issues for dysphagia: modifications involving oral fluids. Journal of Clinical Nursing, 2010. 19(11-12): p. 1618-1624.

24. MacLeod, M., L. Blackie, and S. Humphrey, An investigation into the nutritional efficacy, tolerance and patient acceptance of pre-thickened nutritional supplements in dysphagic patients. Proceedings of the Nutrition Society, 2010. 69: p. E191.

25. Vandewoude, M.F., et al., Fibre-supplemented tube feeding in the hospitalised elderly. Age Ageing, 2005. 34(2): p. 120-4.

26. Cruz-Jentoft, A.J., et al., Compliance with an oral hyperproteic supplement with fibre in nursing home residents. J Nutr Health Aging, 2008. 12(9): p. 669-73.

27. Elia, M., et al., Systematic review and meta-analysis: the clinical and physiological effects of fibre-containing enteral formulae. Aliment Pharmaco Ther, 2008. 27(2): p. 120-45. 